manuscript No.

(will be inserted by the editor)

\title{
A short note on the weak Lefschetz property for Chow groups
}

\section{Robert Laterveer}

\begin{abstract}
Motivated by the Bloch-Beilinson conjectures, we formulate a certain covariant weak Lefschetz property for Chow groups. We prove this property in some special cases, using Kimura's nilpotence theorem.
\end{abstract}

Keywords Algebraic cycles · Chow groups · Finite-dimensional motives · Weak Lefschetz

Mathematics Subject Classification (2010) $14 \mathrm{C} 15 \cdot 14 \mathrm{C} 25 \cdot 14 \mathrm{C} 30$

\section{Introduction}

Let $X$ be a smooth projective variety over $\mathbb{C}$ of dimension $n$. The Chow groups $A^{i} X$ (of codimension $i$ algebraic cycles modulo rational equivalence) are notoriously hard to understand. For instance, the following conjecture dating from 1974 is still completely open for $i>1$ :

Conjecture 1 (Hartshorne [9]) If $Y \subset X$ is a smooth hyperplane section, restriction induces isomorphisms

$$
A^{i} X_{\mathbb{Q}} \cong A^{i} Y_{\mathbb{Q}}
$$

for $2 i<n-1$.

Since this seems a very difficult problem, in this note we try and formulate a covariant weak Lefschetz property for Chow groups and hope this is easier. To emphasize that we consider the Chow groups as a homology theory, we now switch to the notation $A_{i} X=A^{n-i} X$. Let $A_{i}^{h o m}$ and $A_{i}^{A J}$ denote the subgroup of homologically trivial resp. Abel-Jacobi trivial cycles.

To fix ideas, let's now consider $A_{0} X$, the Chow group of 0 -cycles. Since $H^{2 n}(X, \mathbb{Q})$ is one-dimensional, obviously

$$
A_{0} Y_{\mathbb{Q}} \rightarrow A_{0} X_{\mathbb{Q}} / A_{0}^{h o m} X_{\mathbb{Q}}
$$

is surjective for any point $Y$ of $X$-and in particular, for a 0 -dimensional complete intersection $Y \subset X$. The next step is that (by weak Lefschetz applied to $H^{2 n-1}(X, \mathbb{Q})$ )

$$
A_{0} Y_{\mathbb{Q}} \rightarrow A_{0} X_{\mathbb{Q}} / A_{0}^{A J} X_{\mathbb{Q}}
$$

is surjective, for any smooth complete intersection curve $Y \subset X$. Going beyond the Abel-Jacobi map, it is conjectured there is a filtration $F^{*}$ on $A_{0}$, of which the first two steps are $F^{1}=A_{0}^{h o m}$ and $F^{2}=A_{0}^{A J}$ (cf. [12], [19], [20], [26]). One can then ask:

CNRS - IRMA, Université de Strasbourg

7 rue René Descartes

67084 Strasbourg cedex

France

E-mail: laterv@math.unistra.fr 
Question 1 Is it true that

$$
A_{0} Y_{\mathbb{Q}} \rightarrow A_{0} X_{\mathbb{Q}} / F^{\ell+1}
$$

is surjective, for any smooth complete intersection $Y \subset X$ of dimension $\ell$ ?

This question is motivated (pun intended) by the expectation that the quotient $A_{0} X_{\mathbb{Q}} / F^{\ell+1}$ is determined by the cohomology groups $H^{2 n} X, H^{2 n-1} X, \ldots, H^{2 n-\ell} X$. Since the filtration $F^{*}$ only exists conjecturally, this question is not falsifiable. However, it is expected that $F^{\ell+1}$ vanishes exactly when $H^{n} X, \ldots, H^{\ell+1} X$ are supported in codimension 1 . This gives the following conjecture, in which $F^{*}$ does not appear:

Conjecture 2 Let $X$ be a smooth projective variety, and suppose $H^{i}(X, \mathbb{Q})=N^{1} H^{i}(X, \mathbb{Q})$ for $i \in[\ell+1, n]$. Then

$$
A_{0} Y_{\mathbb{Q}} \rightarrow A_{0} X_{\mathbb{Q}}
$$

is surjective, for any smooth complete intersection $Y \subset X$ of dimension $\ell$.

The main result of this note provides a verification of this conjecture in some special cases. As a by-product, we also get the injectivity part of conjecture 1 in these special cases:

Theorem (=Theorem 3) Suppose the Voisin standard conjecture (conjecture 4) holds. Let X be a smooth projective variety of dimension $n$, and suppose

(i) Either the motive of $X$ is finite-dimensional, or Griff ${ }^{n}(X \times X)_{\mathbb{Q}}=0$;

(ii) The Lefschetz standard conjecture $B(X)$ holds;

(iii) There exists $r$ such that $H^{i}(X, \mathbb{Q})=N^{r} H^{i}(X, \mathbb{Q})$ for all $i \in[n-r+1, n]$.

Then for any codimension $r$ smooth complete intersection $Y \subset X$ of class $[Y]=L^{r} \in H^{2 r}(X, \mathbb{Q})$ with $L$ ample, push-forward maps

$$
A_{i}(Y)_{\mathbb{Q}} \rightarrow A_{i}(X)_{\mathbb{Q}}
$$

are surjective for $i<r$. Moreover, restriction maps

$$
A_{A J}^{i}(X)_{\mathbb{Q}} \rightarrow A_{A J}^{i}(Y)_{\mathbb{Q}}
$$

are injective for $i \leq r+1$.

In certain cases some of the hypotheses are automatically satisfied, and the statement simplifies:

Corollary (cf. Corollary 1] Let $X$ be a smooth projective 3fold which is dominated by a product of curves. Suppose

$$
H^{3}(X, \mathbb{Q})=N^{1} H^{3}(X, \mathbb{Q}) .
$$

Then for any smooth ample hypersurface $Y \subset X$, the push-forward map

$$
A_{0}(Y)_{\mathbb{Q}} \rightarrow A_{0}(X)_{\mathbb{Q}}
$$

is surjective, and

$$
A_{A J}^{2}(X)_{\mathbb{Q}} \rightarrow A_{A J}^{2}(Y)_{\mathbb{Q}}
$$

is injective.

Corollary (=Corollary 21) Let $X$ be a product of smooth projective surfaces

$$
X=S_{1} \times \cdots \times S_{m}
$$

where each $S_{j}$ is either a K3 surface of Picard number 19 or 20 , or has $A_{0}^{A J}\left(S_{j}\right)_{\mathbb{Q}}=0$. Suppose at least one $S_{j}$ has $A_{0}^{A J}\left(S_{j}\right)_{\mathbb{Q}}=0$. Then for any smooth ample hypersurface $Y \subset X$, the push-forward map

$$
A_{0}(Y)_{\mathbb{Q}} \rightarrow A_{0}(X)_{\mathbb{Q}}
$$

is surjective, and

$$
A_{A J}^{2}(X)_{\mathbb{Q}} \rightarrow A_{A J}^{2}(Y)_{\mathbb{Q}}
$$

is injective. 
It was already known that in situations like these two corollaries, $A_{0} X_{\mathbb{Q}}$ is supported on some divisor (this follows for instance from [26, Theorem 3.32]); thus, our only contribution is the precision that any ample hypersurface does the job. The injectivity statement, on the other hand, seems to be genuinely new: as far as we know, these are the first examples of varieties with non-trivial $A_{A J}^{2}$ for which this injectivity is known to hold 11 The proof of the theorem is an easy exercice in using the meccano of correspondences; the only "deep" ingredient is Kimura's nilpotence theorem [16].

We end this introduction with a challenge. As is well-known [5], the hypothesis of conjecture 2 is verified when $A_{0} X_{\mathbb{Q}}$ is supported in dimension $\ell$. This gives the following special case of conjecture 2

Conjecture 3 Let $X$ be a smooth projective variety, and suppose $A_{0} X_{\mathbb{Q}}$ is supported on a closed subvariety of dimension $\ell$. Then any smooth complete intersection $Y \subset X$ of dimension $\ell$ supports $A_{0} X_{\mathbb{Q}}$.

This is true for $\ell \leq 1$, but for $\ell>1$ I have no idea how to prove this...

Conventions In this note, the word variety refers to a quasi-projective algebraic variety over $\mathbb{C}$. A subvariety will be a (possibly reducible) reduced subscheme which is equidimensional. The Chow group of $i-d i m e n s i o n a l$ cycles on $X$ is denoted $A_{i} X$; for $X$ smooth of dimension $n$ the notations $A_{i} X$ and $A^{n-i} X$ will be used interchangeably. The Griffiths group Griff ${ }_{i}$ is the group of $i$-dimensional cycles that are homologically trivial modulo algebraic equivalence. In diagrams, we will sometimes write $H^{j} X$ or $H_{j} X$ to designate singular cohomology $H^{j}(X, \mathbb{Q})$ resp. Borel-Moore homology $H_{j}(X, \mathbb{Q})$.

\section{Preliminary}

Definition 1 (Coniveau filtration [4]) Let $X$ be a quasi-projective variety. The coniveau filtration on cohomology and on homology is defined as

$$
\begin{aligned}
N^{c} H^{i}(X, \mathbb{Q}) & =\sum \operatorname{Im}\left(H_{Y}^{i}(X, \mathbb{Q}) \rightarrow H^{i}(X, \mathbb{Q})\right) ; \\
N_{c} H_{i}(X, \mathbb{Q}) & =\sum \operatorname{Im}\left(H_{i}(Z, \mathbb{Q}) \rightarrow H_{i}(X, \mathbb{Q})\right),
\end{aligned}
$$

where $Y$ runs over codimension $\geq c$ subvarieties of $X$, and $Z$ over dimension $\leq c$ subvarieties.

We recall the statement of the "Voisin standard conjecture":

Conjecture 4 (Voisin standard conjecture [25]) Let $X$ be a smooth projective variety, and $Y \subset X$ closed with complement $U$. Then the natural sequence

$$
N_{i} H_{2 i}(Y, \mathbb{Q}) \rightarrow N_{i} H_{2 i}(X, \mathbb{Q}) \rightarrow N_{i} H_{2 i}(U, \mathbb{Q}) \rightarrow 0
$$

is exact for any $i$.

Remark 1 Hodge theory gives an exact sequence

$$
\mathrm{Gr}_{-2 i}^{W} H_{2 i} Y \cap F^{-i} \rightarrow H_{2 i} X \cap F^{-i} \rightarrow \mathrm{Gr}_{-2 i}^{W} H_{2 i} U \cap F^{-i} \rightarrow 0
$$

where $W$ denotes Deligne's weight filtration, and $F$ the Hodge filtration on $H_{*}(-, \mathbb{C})$. Hence if the Hodge conjecture (that is, its homology version for singular varieties [11]) is true, then conjecture 4is true.

What's more, this conjecture fits in very neatly with the classical standard conjectures: Voisin shows that conjecture 4 plus the algebraicity of the Künneth components of the diagonal is equivalent to the Lefschetz standard conjecture [25, Proposition 1.6].

Remark 2 Conjecture 4 is obviously true for $i \leq 1$ (this follows from the truth of Hodge conjecture for curve classes), and for $i \geq \operatorname{dim} Y-1$ (where it follows from the Hodge conjecture for divisors).

The main ingredient used in this note is Kimura's nilpotence theorem:

\footnotetext{
1 This is not strictly true: indeed, [7] Corollary 5] gives non-trivial examples of varieties where the injectivity part of conjecture 1 is verified.
} 
Theorem 1 (Kimura [14]) Let $X$ be a smooth projective variety of dimension $n$ with finite-dimensional motive. Let $\Gamma \in A^{n}(X \times X)_{\mathbb{Q}}$ be a correspondence which is homologically trivial. Then there is $N \in \mathbb{N}$ such that

$$
\Gamma^{\circ N}=0 \quad \in A^{n}(X \times X)_{\mathbb{Q}} .
$$

Remark 3 We refer to [14], [1], [20] for the definition of finite-dimensional motive. Conjecturally, any variety has finite-dimensional motive [14]. What mainly concerns us in the scope of this note, is that there are quite a few examples which are known to have finite-dimensional motive: varieties dominated by products of curves [14], $K 3$ surfaces with Picard number 19 or 20 [21], any surface with vanishing geometric genus for which Bloch's conjecture has been verified [8, Theorem 2.11], 3folds with nef tangent bundle [10], certain 3folds of general type [22, Section 8].

There is also the following nilpotence result, which predates Kimura's theorem:

Theorem 2 (Voisin [24], Voevodsky [23]) Let $X$ be a smooth projective algebraic variety of dimension $n$, and $\Gamma \in A^{n}(X \times X)_{\mathbb{Q}}$ a correspondence which is algebraically trivial. Then there is $N \in \mathbb{N}$ such that

$$
\Gamma^{\circ N}=0 \quad \in A^{n}(X \times X)_{\mathbb{Q}}
$$

\section{Main}

We now proceed with the proof of the main result of this note:

Theorem 3 Suppose the Voisin standard conjecture holds. Let $X$ be a smooth projective variety of dimension $n$, and suppose

(i) Either the motive of $X$ is finite-dimensional, or Griff ${ }^{n}(X \times X)_{\mathbb{Q}}=0$;

(ii) The Lefschetz standard conjecture $B(X)$ holds;

(iii) $H^{i}(X, \mathbb{Q})=N^{r} H^{i}(X, \mathbb{Q})$ for all $i \in[n-r+1, n]$.

Then for any codimension $r$ smooth complete intersection $Y \subset X$ of class $[Y]=L^{r} \in H^{2 r}(X, \mathbb{Q})$ with $L$ ample, push-forward maps

$$
A_{i}(Y)_{\mathbb{Q}} \rightarrow A_{i}(X)_{\mathbb{Q}}
$$

are surjective for $i<r$. Moreover, restriction maps

$$
A_{A J}^{i}(X)_{\mathbb{Q}} \rightarrow A_{A J}^{i}(Y)_{\mathbb{Q}}
$$

are injective for $i \leq r+1$.

In certain cases, some of the hypotheses can be removed:

Corollary 1 Let $X$ be a smooth projective variety of dimension $n \leq 3$, and suppose

(i) Either the motive of $X$ is finite-dimensional, or Griff $(X \times X)_{\mathbb{Q}}=0$;

(ii) $H^{n}(X, \mathbb{Q})=N^{1} H^{n}(X, \mathbb{Q})$.

Then for any smooth ample hypersurface $Y \subset X$, push-forward maps

$$
A_{0}(Y)_{\mathbb{Q}} \rightarrow A_{0}(X)_{\mathbb{Q}}
$$

are surjective, and restriction

$$
A_{A J}^{2}(X)_{\mathbb{Q}} \rightarrow A_{A J}^{2}(Y)_{\mathbb{Q}}
$$

is injective.

Corollary 2 Let $X$ be a product of smooth projective surfaces

$$
X=S_{1} \times \cdots \times S_{m}
$$

where each $S_{j}$ is either a K3 surface of Picard number 19 or 20 , or has $A_{0}^{A J}\left(S_{j}\right)_{\mathbb{Q}}=0$. 
(i) Suppose at least one $S_{j}$ has $A_{0}^{A J}\left(S_{j}\right)_{\mathbb{Q}}=0$. Then for any smooth ample hypersurface $Y \subset X$,

$$
A_{0}(Y)_{\mathbb{Q}} \rightarrow A_{0}(X)_{\mathbb{Q}}
$$

is surjective, and

$$
A_{A J}^{2}(X)_{\mathbb{Q}} \rightarrow A_{A J}^{2}(Y)_{\mathbb{Q}}
$$

is injective.

(ii) Suppose there are at least 4 surfaces $S_{j}$ with $A_{0}^{A J}\left(S_{j}\right)_{\mathbb{Q}}=0$. Let $Y \subset X$ be a codimension 2 complete intersection of class $[Y]=L^{2} \in H^{4}(X, \mathbb{Q})$ with L ample. Then

$$
A_{i}(Y)_{\mathbb{Q}} \rightarrow A_{i}(X)_{\mathbb{Q}}
$$

is surjective for $i \leq 1$, and

$$
A_{A J}^{i}(X)_{\mathbb{Q}} \rightarrow A_{A J}^{i}(Y)_{\mathbb{Q}}
$$

is injective for $i \leq 3$.

Proof (of theorem 3) Let $\tau: Y \hookrightarrow X$ be a smooth complete intersection of class $L^{r}$ as in the statement of the theorem. Let

$$
L^{j}: H^{i} X(\mathbb{Q}) \rightarrow H^{i+2 j}(X, \mathbb{Q})
$$

denote the result of cupping with a power of $L$; we use the same notation $L^{j}$ for the correspondence inducing this action. Since $B(X)$ is true, for any $i<n$ there exists a correspondence $C_{i} \in A^{i}(X \times X)_{\mathbb{Q}}$ inducing an isomorphism

$$
\left(C_{i}\right)_{*}: H^{2 n-i}(X, \mathbb{Q}) \stackrel{\cong}{\rightrightarrows} H^{i}(X, \mathbb{Q})
$$

that is inverse to $L^{n-i}$.

$B(X)$ being true, the Künneth components $\pi_{i}$ of the diagonal of $X$ are algebraic [16]. Since $B(X)$ implies $B(Y)$ [16], the same holds for the Künneth components $\pi_{i}^{Y}$ of $Y$. We now proceed to relate them:

Lemma 1 For each $i \leq n-r$, define

$$
\Pi_{i}:=\left(C_{i}\right) \circ\left(L^{n-i-r}\right) \circ\left((\tau \times \tau)_{*}\left(\pi_{i}^{Y}\right)\right) \in A^{n}(X \times X, \mathbb{Q}) .
$$

Then for each $i \leq n-r$, we have equality

$$
\Pi_{i}=\pi_{i} \in H^{2 n}(X \times X, \mathbb{Q}) .
$$

Proof We consider the action on $H^{j}(X, \mathbb{Q})$. There is a factorization

$$
\begin{array}{ccc}
H^{j} X \stackrel{\left((\tau \times \tau)_{*}\left(\pi_{i}^{Y}\right)\right)_{*}}{\longrightarrow} & H^{j+2 r} X \stackrel{L^{n-i-r}}{\longrightarrow} H^{2 n-2 i+j} X \stackrel{\left(C_{i}\right)_{*}}{\longrightarrow} H^{j} X \\
\downarrow & \uparrow \\
H^{j} Y \stackrel{\left(\pi_{i}^{Y}\right)_{*}}{\longrightarrow} & H^{j} Y
\end{array}
$$

Hence, if $j \neq i$ then

$$
\left(\Pi_{i}\right)_{*} H^{j} X=0,
$$

and for $j=i$ we have

$$
\Pi_{i}=\left(C_{i}\right) \circ\left(L^{n-i-r}\right) \circ\left(L^{r}\right)=\mathrm{id}: H^{i} X \rightarrow H^{i} X .
$$

It follows that for any variety $Z$, the action of $\Pi_{i}$ on $H^{j}(X \times Z)$ is projection on $H^{i} X \otimes H^{j-i} Z$; thus by Manin's identity principle, $\Pi_{i}$ and $\pi_{i}$ coincide as homological correspondences.

Lemma 2 For each $i \leq n-r$, and each $j<r$, we have

$$
\left(\Pi_{i}\right)_{*} A_{j} X_{\mathbb{Q}}=0 .
$$


Proof For any correspondence $C \in A^{n-r}(Y \times Y)_{\mathbb{Q}}$, there is a factorization

$$
\begin{array}{ccc}
A_{j} X_{\mathbb{Q}} & \stackrel{\left((\tau \times \tau)_{*} C\right)_{*}}{\longrightarrow} & A_{j-r} X_{\mathbb{Q}} \\
\downarrow & \uparrow \\
A_{j-r} Y_{\mathbb{Q}} & \stackrel{C_{*}}{\longrightarrow} & A_{j-r} Y_{\mathbb{Q}}
\end{array}
$$

In particular, taking $C=\pi_{i}^{Y}$, we see that the action of $(\tau \times \tau) *\left(\pi_{i}^{Y}\right)$ on $A_{j} X_{\mathbb{Q}}$ factors over $A_{j-r} Y_{\mathbb{Q}}$, hence is 0 for $j<r$.

Lemma 3 Let $^{t} \Pi_{i}$ denote the transpose of $\Pi_{i}$. For each $i \leq n-r$, and each $j$, we have

$$
\left({ }^{t} \Pi_{i}\right)_{*} A_{j} X_{\mathbb{Q}} \subset \operatorname{Im}\left(A_{j} Y_{\mathbb{Q}} \rightarrow A_{j} X_{\mathbb{Q}}\right) .
$$

Moreover, for each $j \leq r+1$, we have

$$
\left({ }^{t} \Pi_{i}\right)_{*} A_{A J}^{j} X_{\mathbb{Q}}=0
$$

Proof It is immediate from the definition that

$$
{ }^{t} \Pi_{i}=\left((\tau \times \tau) *\left({ }^{t} \pi_{i}^{Y}\right)\right) \circ{ }^{t}\left(L^{n-i-r}\right) \circ{ }^{t} C_{i} \in A^{n}(X \times X)_{\mathbb{Q}}
$$

Using the same diagram as in the proof of lemma2, one can find a factorization

$$
\begin{aligned}
& A_{j} X_{\mathbb{Q}} \stackrel{\left({ }^{t}\left(L^{n-i-r}\right) \circ^{t} C_{i}\right)_{*}}{\longrightarrow} \quad A_{j+r} X_{\mathbb{Q}} \stackrel{\left((\tau \times \tau)_{*}\left({ }^{t} \pi_{i}^{Y}\right)\right)_{*}}{\longrightarrow} A_{j} X_{\mathbb{Q}} \\
& A_{j} Y_{\mathbb{Q}} \stackrel{{ }^{t}\left(\pi_{i}^{Y}\right)_{*}}{\longrightarrow} \quad A_{j} Y_{\mathbb{Q}}
\end{aligned}
$$

and the lemma is proven.

By hypothesis (iii), we have

$$
H^{i}(X, \mathbb{Q})=N^{r} H^{i}(X, \mathbb{Q}) \forall n-r<i \leq n .
$$

Applying hard Lefschetz, one finds

$$
H^{i}(X, \mathbb{Q})=N^{r} H^{i}(X, \mathbb{Q}) \forall n-r<i<n+r .
$$

This means that in the range $n-r<i<n+r$, the Künneth component $\pi_{i}$ is supported in codimension $r$. That is, there exists a subvariety $Z \subset X$ of codimension $r$, such that for each $n-r<i<n+r$, $\pi_{i}$ goes to 0 under the restriction

$$
H^{2 n}(X \times X, \mathbb{Q}) \rightarrow H^{2 n}((X \times X) \backslash(Z \times Z), \mathbb{Q}) .
$$

Using the Voisin standard conjecture (conjecture 4), this implies the existence of an algebraic cycle $P_{i}^{\prime} \in A_{n}(Z \times$ $Z)_{\mathbb{Q}}$ such that (denoting by $P_{i}$ the push-forward of $P_{i}^{\prime}$ to $X \times X$ ) we have

$$
P_{i}=\pi_{i} \in H^{2 n}(X \times X, \mathbb{Q}) \forall n-r<i<n+r .
$$

Lemma 4 For any $i \in[n-r+1, n+r-1]$, and any $j<r$, we have

$$
\left(P_{i}\right)_{*} A_{j} X_{\mathbb{Q}}=0
$$

Moreover, for any $j \leq r+1$, we have

$$
\left(P_{i}\right)_{*} A_{A J}^{j} X_{\mathbb{Q}}=0
$$


Proof Let $\psi: Z \rightarrow X$ denote the inclusion, so $P_{i}=(\psi \times \psi)_{*}\left(P_{i}^{\prime}\right)$. Similar to lemma 2] there is a factorization

$$
\begin{array}{ccc}
A_{j} X_{\mathbb{Q}} & \stackrel{\left(P_{i}\right)_{*}}{\longrightarrow} A_{j} X_{\mathbb{Q}} \\
\downarrow & & \uparrow \\
A_{j-r} Z_{\mathbb{Q}} & \stackrel{\left(P_{i}^{\prime}\right)_{*}}{\longrightarrow} A_{j} Z_{\mathbb{Q}} .
\end{array}
$$

That is, the action of $P_{i}$ in the indicated range factors over groups that vanish for dimension reasons and the lemma follows.

Putting together the various parts, we find a decomposition of the diagonal

$$
\Delta=\sum_{i=0}^{n-r} \Pi_{i}+\sum_{i=n-r+1}^{n+r-1} P_{i}+\sum_{i=0}^{n-r}{ }^{t} \Pi_{i} \in H^{2 n}(X \times X, \mathbb{Q}) .
$$

This is an equality of cycles modulo homological equivalence. Now, applying Kimura's nilpotence theorem (theorem 11, we get that there exists $N$ such that

$$
\left(\Delta-\sum_{i=1}^{n-r} \Pi_{i}-\sum_{i=n-r+1}^{n+r-1} P_{i}-\sum_{i=1}^{n-r}{ }^{t} \Pi_{i}\right)^{\circ N}=0 \in A^{n}(X \times X)_{\mathbb{Q}}
$$

Developing this expression (and noting that $\Delta^{\circ N}=\Delta$ ), we find

$$
\Delta=\sum_{j} Q_{j} \in A^{n}(X \times X)_{\mathbb{Q}},
$$

where each $Q_{j}$ is a composition of elements $\Pi_{\ell}$ and $P_{\ell^{\prime}}$ and ${ }^{t} \Pi_{\ell^{\prime \prime}}$. Let $Q_{j}^{0}$ denote the "tail element" of $Q_{j}$, i.e. we write

$$
Q_{j}=Q_{j}^{0} \circ Q_{j}^{1} \circ \cdots \circ Q_{j}^{N^{\prime}} \in A^{n}(X \times X)_{\mathbb{Q}},
$$

with $Q_{j}^{0} \neq \Delta$ (so that $\left.N^{\prime} \leq N\right)$.

Let's consider the action of $Q_{j}$ on $A_{i} X_{\mathbb{Q}}$, for $i<r$ :

If $Q_{j}^{0}$ is a $\Pi_{\ell}$ (for some $\ell \in[0, n-r]$ ), it follows from lemma 2 that

$$
\left(Q_{j}\right)_{*}\left(A_{i} X_{\mathbb{Q}}\right)=0 .
$$

Likewise, if $Q_{j}^{0}$ is of the form $P_{\ell}$ (for some $n-r<\ell<n+r$ ), then applying lemma 4, we find again

$$
\left(Q_{j}\right)_{*}\left(A_{i} X_{\mathbb{Q}}\right)=0 .
$$

Finally, if $Q_{j}^{0}$ is of the form ${ }^{t} \Pi_{\ell}$ (for some $\ell \in[0, n-r]$ ), it follows from lemma 3 that

$$
\left(Q_{j}\right)_{*}\left(A_{i} X_{\mathbb{Q}}\right) \subset \operatorname{Im}\left(A_{i} Y_{\mathbb{Q}} \rightarrow A_{i} X_{\mathbb{Q}}\right) .
$$

Since $\Delta$ acts as the identity, we conclude that for $i<r$, push-forward

$$
A_{i} Y_{\mathbb{Q}} \rightarrow A_{i} X_{\mathbb{Q}}
$$

is surjective.

The argument for the injectivity statement is similar: we consider the action of $\Delta=\sum_{j} Q_{j}$ on $A_{A J}^{i} X_{\mathbb{Q}}$ for $i \leq r+1$. If $Q_{j}$ is such that its "head" $Q_{j}^{N^{\prime}}$ is of type ${ }^{t} \Pi_{\ell}$ or $P_{\ell}$, then $Q_{j}$ does not act (by lemma $3 \mathrm{resp}$. lemma 4). It follows that we can write

$$
A_{A J}^{i} X_{\mathbb{Q}}=\Delta_{*} A_{A J}^{i} X_{\mathbb{Q}}=\left(\sum \text { something } \circ(\tau \times \tau)_{*}(\text { something })\right)_{*} A_{A J}^{i} X_{\mathbb{Q}}
$$

the injectivity is then obvious. 
Finally, if the hypothesis in (i) of the theorem is that

$$
\operatorname{Griff}^{n}(X \times X)_{\mathbb{Q}}=0
$$

the proof goes as follows: the decomposition of $\Delta$ is now an equality modulo algebraic equivalence (since by hypothesis, algebraic and homological equivalence coincide on $X \times X$ ). Then, instead of applying Kimura's theorem, we apply the Voisin/Voevodsky nilpotence theorem (theorem 2). The rest of the proof is verbatim the same.

Proof (of corollary 1) In case $n=2$, we know $B(X)$ holds since it holds for any surface [16]. The Voisin standard conjecture is used to get that some Hodge classes in $H_{4}(Z \times Z, \mathbb{Q})$ are algebraic, where $\operatorname{dim} Z=1$; this is trivially true.

Next, the case $n=3$. Under the hypothesis $H^{3} X=N^{1} H^{3} X, X$ is "motivated by a surface" in the sense of [2], so $B(X)$ is known to hold [2]. The Voisin standard conjecture is only used to get that some Hodge classes in $H_{6}(Z \times Z, \mathbb{Q})$ are algebraic, where $\operatorname{dim} Z=2$; this is OK by the Hodge conjecture for divisors (remark 2).

Proof (of corollary 2) As we noted in remark 3] it follows from work of Pedrini [21] and Guletskiǔ-Pedrini [8] that the $S_{j}$ have finite-dimensional motive. Hence $X$ has finite-dimensional motive. We also know $B(X)$ is true since the Lefschetz standard conjecture is true for all surfaces [16].

In case (i), since there is at least one surface with $H^{2}\left(S_{j}\right)=N^{1}$, we obviously have

$$
H^{2 m}(X, \mathbb{Q})=N^{1} H^{2 m}(X, \mathbb{Q}) .
$$

The corollary now follows from theorem 3 , note that we don't need to assume the Voisin standard conjecture, since we can find cycles $P_{i}^{\prime}$ by using the Hodge conjecture on the surfaces with vanishing geometric genus.

In case (ii), the assumptions imply

$$
\begin{aligned}
H^{2 m}(X, \mathbb{Q}) & =N^{2} H^{2 m}(X, \mathbb{Q}) ; \\
H^{2 m-1}(X, \mathbb{Q}) & =N^{2} H^{2 m-1}(X, \mathbb{Q}),
\end{aligned}
$$

and we again apply theorem 3

Remark 4 The hypothesis on $\operatorname{Griff}^{n}(X \times X)$ in theorem 3 is mainly of theoretical interest, and not practically useful. Indeed, there are precise conjectures predicting when Griffiths groups should vanish [13]; for instance, if $X$ is a 4fold with $h^{2,0}=h^{4,0}=h^{3,0}=h^{2,1}=0$, [13, Corollary 6.8] implies that if the Bloch-Beilinson conjectures are true then

$$
\operatorname{Griff}^{4}(X \times X)_{\mathbb{Q}}=0 .
$$

Unfortunately, no non-trivial examples seem to be known. Specifically, I am not aware of any example of a variety $X$ of dimension $n$ that satisfies $\operatorname{Griff}^{n}(X \times X)_{\mathbb{Q}}=0$, but not $A_{A J}^{i} X_{\mathbb{Q}}=0 \forall i$.

Remark 5 In [17], I study a certain hard Lefschetz property for Chow groups. Using arguments similar to the present note, this hard Lefschetz property can be proven in some special cases [17].

Acknowledgements This note was written while preparing for the Strasbourg "groupe de travail" based on the monograph [26]. I wish to thank all the participants of this groupe de travail for the very pleasant and stimulating atmosphere.

\section{References}

1. Y. André, Motifs de dimension finie (d'après S.-I. Kimura, P. O’Sullivan...), Séminaire Bourbaki. Vol. 2003/2004. Astérisque 299 Exp. No. 929, viii, 115-145,

2. D. Arapura, Varieties with very little transcendental cohomology, in: Motives and algebraic cycles (R. de Jeu and J. Lewis, eds.), Fields Institute Communications, Amer. Math. Soc., Providence 2009,

3. D. Arapura, Motivation for Hodge cycles, Advances in Math. vol. 207 (2006),

4. S. Bloch and A. Ogus, Gersten's conjecture and the homology of schemes, Ann. Sci. Ecole Norm. Sup. 4 (1974), 181—202, 
5. S. Bloch and V. Srinivas, Remarks on correspondences and algebraic cycles, American Journal of Mathematics Vol. 105, No 5 (1983), 1235-1253,

6. W. Fulton, Intersection theory, Springer-Verlag Berlin Heidelberg New York 1984,

7. B. Fu, Remarks on hard Lefschetz conjectures on Chow groups, Science China Mathematics Vol. 53 No 1 (2010), 105-114,

8. V. Guletskil and C. Pedrini, The Chow motive of the Godeaux surface, in: Algebraic Geometry, a volume in memory of Paolo Francia (M.C. Beltrametti, F. Catanese, C. Ciliberto, A. Lanteri and C. Pedrini, editors), Walter de Gruyter, Berlin New York, 2002,

9. R. Hartshorne, Equivalence relations on algebraic cycles and subvarieties of small codimension, in: Algebraic geometry, Arcata 1974, Proc. Symp. Pure Math. Vol. 29, Amer. Math. Soc., Providence 1975,

10. J. Iyer, Murre's conjectures and explicit Chow-Künneth projectors for varieties with a nef tangent bundle, Transactions of the Amer. Math. Soc. 361 (2008), 1667-1681,

11. U. Jannsen, Mixed motives and algebraic K-theory, Springer Lecture Notes in Mathematics 1400 (1990),

12. U. Jannsen, Motivic sheaves and filtrations on Chow groups, in: Motives (Jannsen et alii, eds.), Proceedings of Symposia in Pure Mathematics Vol. 55 (1994), Part 1,

13. U. Jannsen, Equivalence relations on algebraic cycles, in: The arithmetic and geometry of algebraic cycles (B. Gordon et alii, eds.), Banff Conf. 1998, Kluwer,

14. S. Kimura, Chow groups are finite dimensional, in some sense, Math. Ann. 331 (2005), 173 —201,

15. S. Kleiman, Algebraic cycles and the Weil conjectures, in: Dix exposés sur la cohomologie des schémas, North-Holland Amsterdam, 1968, 359-386,

16. S. Kleiman, The standard conjectures, in: Motives (Jannsen et alii, eds.), Proceedings of Symposia in Pure Mathematics Vol. 55 (1994), Part 1,

17. R. Laterveer, A brief note concerning hard Lefschetz for Chow groups, to appear in Canadian Math. Bulletin,

18. D. Mumford, Rational equivalence of 0-cycles on surfaces, J. Math. Kyoto Univ. Vol. 9 No 2 (1969), 195-204,

19. J. Murre, On a conjectural filtration on the Chow groups of an algebraic variety, Indag. Math. (N.S.) 4 (1993), 177-201,

20. J. Murre, J. Nagel and C. Peters, Lectures on the theory of pure motives, Amer. Math. Soc. University Lecture Series 61, 2013,

21. C. Pedrini, On the finite dimensionality of a $K 3$ surface, Manuscripta Mathematica 138 (2012), 59—72,

22. C. Vial, Chow-Künneth decomposition for 3- and 4-folds fibred by varieties with trivial Chow group of zero-cycles, J. Algebraic Geom. 24 (2015), 51-80,

23. V. Voevodsky, A nilpotence theorem for cycles algebraically equivalent to zero, Internat. Math. Research Notices 4 (1995), 187-198,

24. C. Voisin, Remarks on zero-cycles on self-products of varieties, in: Moduli of vector bundles (Proceedings of the Taniguchi Congress), Maruyama Ed., Decker 1994,

25. C. Voisin, The generalized Hodge and Bloch conjectures are equivalent for general complete intersections, Annales scientifiques de l'ENS 46, fascicule 3 (2013), 449-475,

26. C. Voisin, Chow Rings, Decomposition of the Diagonal, and the Topology of Families, Princeton University Press, Princeton and Oxford, 2014. 